\section{Utilização de medicamentos e fatores associados: um estudo de base populacional no Município de Campinas, São Paulo, Brasil}

\author{
Use of medication and associated factors: \\ a population-based study in Campinas, \\ São Paulo State, Brazil
}

\footnotetext{
1 Faculdade de Ciências Médicas, Universidade Estadual de Campinas, Campinas, Brasil.

2 Faculdade de Saúde Pública, Universidade de São Paulo, São Paulo, Brasil.

3 Faculdade de Medicina, Universidade de São Paulo, São Paulo, Brasil. ${ }^{4}$ Faculdade de Medicina de Botucatu, Universidade Estadual Paulista Júlio de Mesquita Filho, Botucatu, Brasil.

Correspondência K. S. Costa

Faculdade de Ciências Médicas, Universidade Estadual de Campinas. Cidade Universitária Barão Geraldo, C. P. 6111, Campinas, $S P$

13083-970, Brasil. karensc@fcm.unicamp.br
}

\begin{abstract}
This article analyzes prevalence rates in the use of medication according to demographic, socioeconomic, and health-related behavioral variables through a population-based crosssectional study of individuals 18 years and older ( $n=941)$ in Campinas, São Paulo State, Brazil. The study used multistage sampling, both stratified and cluster. Chi-square test was performed, and adjusted prevalence ratios were estimated by gender and age, both with 95\% confidence intervals. A Poisson multiple regression model was developed, and the following factors were associated with use of medication: female gender, age 40 and over, reported illness in the previous two weeks, and number of chronic diseases. The most widely consumed drugs were for the cardiovascular and nervous systems, besides herbal remedies. Prevalence of medication in Campinas was lower than in most studies. Local health surveys could help identify drug use patterns and guarantee more appropriate interventions for pharmaceutical care policy.
\end{abstract}

Drug Utilization; Pharmacoepidemiology; Prevalence

\author{
Karen Sarmento Costa 1 \\ Marilisa Berti de Azevedo Barros 1 \\ Priscila Maria Stolses Bergamo Francisco 1 \\ Chester Luis Galvão César 2 \\ Moisés Goldbaum 3 \\ Luana Carandina 4
}

\section{Introdução}

Os medicamentos são importantes instrumentos terapêuticos utilizados no processo saúde/doença, sendo responsáveis por parte significativa do aumento da expectativa e da qualidade de vida da população ${ }^{1}$.

A utilização dos medicamentos é influenciada pela estrutura demográfica, fatores socioeconômicos, comportamentais e culturais, pelo perfil de morbidade, pelas características do mercado farmacêutico e das políticas governamentais dirigidas ao setor 1,2,3,4. Esse conjunto de fatores precisa ser investigado para permitir a melhor compreensão do papel dos medicamentos no contexto da saúde pública.

Em países de baixa e média rendas, em geral, não existem sistemas informatizados que integram os dados acerca dos medicamentos ${ }^{5}$. No caso do Brasil, não há informações sobre o consumo de medicamentos geradas com base em banco de dados de abrangência nacional. Portanto, essas informações precisam ser coletadas utilizando-se outras fontes que permitam aos gestores entender a utilização dos medicamentos e seus determinantes, e poderem promover o aprimoramento das políticas de saúde 6 . Essa situação faz com que o Ministério da Saúde, responsável pela Política de Assistência Farmacêutica e outros órgãos fomentadores de pesquisa, priorizem na agenda a realização de estudos 
nacionais que investiguem questões relativas ao acesso e uso de medicamentos no país.

Reconhecemos que os inquéritos de base populacional são meios apropriados para o monitoramento do estado de saúde e do desempenho dos sistemas de saúde 7. Constituem instrumentos importantes para obtenção de informações relativas à utilização de medicamentos pelos diferentes segmentos sociais da população e também sobre o tipo de medicamento consumido, o perfil demográfico de quem os consome, como e para qual finalidade os fármacos foram usados. Os resultados são úteis para o planejamento das Políticas de Assistência Farmacêutica, de regulação sanitária e para a promoção do uso racional de medicamentos 6 .

Em geral esses estudos de base populacional têm apontado para um maior consumo de medicamentos entre as mulheres $2,3,4,8$, com o aumento da idade $2,3,4$, entre aqueles com maior poder aquisitivo 2,3,4,5,9, entre os mais escolarizados 3,10 , com maior número de doenças crônicas $2,4,9$, entre outras categorias.

Considerando que os padrões de uso de medicamentos diferem entre regiões e se modificam no decorrer do tempo em função das mudanças do perfil saúde/doença e das políticas de saúde implementadas, são necessárias investigações locais que permitam identificar, monitorar e produzir informações sobre uso de medicamento pela população. Nesse sentido, o presente estudo tem por objetivo analisar a prevalência da utilização de medicamentos pela população adulta do Município de Campinas, São Paulo, e os fatores determinantes deste uso, segundo as variáveis demográficas, socioeconômicas, comportamentos relacionados à saúde e morbidade.

\section{Métodos}

Trata-se de um estudo transversal de base populacional que utilizou dados do Inquérito Multicêntrico de Saúde no Estado de São Paulo (ISA-SP) 11, realizado em quatro áreas do estado. Para o presente estudo foi feito um recorte desse Inquérito e analisados os dados da população com idade igual ou superior a 18 anos (941 indivíduos), não institucionalizada e residente em área urbana de Campinas.

Os participantes do inquérito foram selecionados por meio de amostragem probabilística, estratificada, por conglomerados e obtida em dois estágios. No primeiro, os setores censitários foram agrupados em três estratos segundo o porcentual de chefes de família com nível universitário: menos de $5 \%$, de $5 \%-25 \%$ e com mais de $25 \%$. Foram selecionados 10 setores de cada estrato de forma sistemática e com probabilidade de inclusão proporcional ao tamanho, expresso pelo número de domicílios existentes no setor, de acordo com a Contagem Populacional de 1996 do Instituto Brasileiro de Geografia e Estatística (IBGE). No segundo estágio, uma amostra sistemática simples de domicílios foi sorteada em cada setor censitário, de forma a obter um número mínimo de 200 pessoas para cada domínio de idade e sexo (menores de 1 ano; de 1-11 anos; homens e mulheres nas faixas etárias de 12-19 anos, 2059 e 60 anos ou mais de idade, separadamente). Esperando-se uma taxa de $20 \%$ de perda, foram sorteados 250 indivíduos de cada domínio.

No cálculo do tamanho da amostra foi considerada a estimativa de uma proporção de 0,50 , com nível de 95\% de confiança, erro máximo de 0,10 e efeito de delineamento igual a 2 . Detalhes do procedimento de amostragem do inquérito encontram-se publicados 12 .

As informações foram obtidas por meio de entrevistas domiciliares, realizadas por entrevistadores treinados, no período de abril de 2001 a agosto de 2002, respondidas diretamente pelos moradores sorteados. O questionário aplicado foi estruturado com a maioria das questões fechadas e organizado em blocos temáticos: condições de vida, estilo de vida, percepção e qualidade de saúde, morbidade crônica referida, uso de serviços de saúde e consumo de medicamentos.

\section{Variáveis analisadas neste estudo}

Sobre os medicamentos, as variáveis analisadas foram: número de medicamentos, número médio de medicamentos utilizados, uso de ao menos um medicamento no período recordatório definido e o tipo de medicamento.

A variável dependente do estudo foi o uso de pelo menos um medicamento nos três dias anteriores à entrevista, obtida pela pergunta: "Usou algum medicamento nos últimos três dias?”.

Toda vez que o entrevistado referia ter feito uso de algum medicamento nos últimos três dias era solicitado que ele apresentasse a embalagem e/ou a prescrição médica, a fim de minimizar possíveis erros na anotação dos dados pelo entrevistador. $\mathrm{Na}$ ausência da embalagem e/ou prescrição, era anotado o nome comercial do medicamento ou o princípio ativo segundo o relato do entrevistado.

Para a identificação do princípio ativo foi utilizado o Dicionário de Especialidades Farmacêuticas (DEF/2001/2002) 13, e a codificação dos medicamentos foi feita de acordo com o Anatomical Therapeutic Chemical Index (ATC/DDD Index) ${ }^{14}$. Nessa classificação, são considerados o órgão ou sistema em que o medicamento atua 
e as propriedades terapêuticas e farmacológicas. Para os medicamentos cujos nomes o entrevistado não soube referir foi atribuído um código de não identificado; para os produtos que não constavam na ATC foram criados códigos para identificá-los; e para aqueles que não apresentavam um código especificado na ATC foi utilizado o código até o limite que possibilitasse identificar o grupo, a classe ou a ação terapêutica.

Foram analisados quatro conjuntos de variáveis independentes: características demográficas, socioeconômicas, comportamentos relacionados à saúde, e condições de saúde e morbidade.

Sociodemográficas: sexo (feminino e masculino); idade (18-29, 30-39, 40-49, 50-59, 60-69, 70 anos ou mais); situação conjugal (casado/unido, solteiro e separado/viúvo); cor/etnia autorreferida (branca, preta/parda, outras); religião (católico, evangélico e outras religiões, ou sem religião).

Socioeconômicas: escolaridade e renda mensal familiar per capita em salários mínimos. Para o cálculo da renda per capita foram utilizados como base os valores dos salários mínimos vigentes no país à época da pesquisa.

Comportamentos relacionados à saúde: hábito de fumar (fumante, não-fumante, ex-fumante), frequência semanal de ingestão de bebida alcoólica, dependência alcoólica avaliada por meio do teste CAGE 15 , e prática de atividade física em contexto de lazer (obtida pela pergunta: "Pratica regularmente, pelo menos uma vez por semana, algum esporte ou exercício físico? Se sim, qual?”).

Condições de saúde e morbidade: autoavaliação da saúde, hospitalização no último ano, índice de massa corporal (IMC $=\mathrm{kg} / \mathrm{m}^{2}$ ) calculado com dados de peso e altura referidos, transtorno mental comum avaliado com base no Self Reporting Questionnaire (SRQ-20) com ponto de corte $7 / 816$, presença de morbidade nos últimos 15 dias (sim ou não) e número de doenças crônicas por meio do checklist das seguintes doenças: hipertensão, diabetes, doença da pele, alergia, anemia, doença de coluna/costas, artrite/reumatismo/ artrose, doença renal crônica, acidente vascular cerebral, depressão/ansiedade/problemas emocionais, enxaqueca/dor de cabeça, osteoporose, cirrose, epilepsia, doença de Chagas, hanseníase, tuberculose, esquistossomose, câncer, doença do coração, doença crônica do pulmão, doença digestiva crônica, outros.

\section{Análise dos dados}

Foi utilizado o comando svy do programa Stata 8.0 (Stata Corp., College Station, Estados Unidos), que permite considerar a estratificação, a existência de conglomerados, as probabilidades desiguais de seleção das unidades amostrais e ajustes para não-resposta.

Foram estimadas as prevalências de uso de pelo menos um medicamento, nos três dias anteriores à entrevista, segundo as variáveis independentes e os números médios de medicamentos utilizados e os respectivos intervalos de $95 \%$ de confiança (IC95\%), segundo idade e sexo. Usamos o teste qui-quadrado para verificar a associação estatística entre a variável dependente e as independentes. Estimou-se as razões de prevalência ajustadas por sexo e idade e os respectivos IC95\%, utilizando-se regressão múltipla de Poisson.

Foi desenvolvido um modelo de regressão múltipla de Poisson para ajuste de variáveis de confundimento. Foram introduzidas as variáveis que mostraram associação com a variável dependente no nível de significância de $20 \%(\mathrm{p}<0,20)$, permanecendo no modelo aquelas com $\mathrm{p}<0,05$.

O presente estudo foi aprovado pelo Comitê de Ética em Pesquisa da Faculdade de Ciências Médicas da Universidade Estadual de Campinas (UNICAMP), sob o parecer no. 369/2000.

\section{Resultados}

O estudo incluiu 941 pessoas, de 18 anos ou mais, sendo $51,1 \%$ (481) homens. Da população estudada, 51,5\% não fizeram uso de medicamentos nos três dias que antecederam a entrevista, sendo este porcentual maior entre os homens. Conforme apresentado na Tabela 1, o número médio de medicamentos utilizados foi de 1,81, sendo que houve aumento de acordo com a idade em ambos os sexos, sendo sempre maior entre as mulheres e atingindo uma média de 3,55 medicamentos dentre mulheres com 70 anos ou mais.

\section{Tabela 1}

Número médio de medicamentos utilizados nos últimos três dias, segundo sexo, pela população de 18 anos ou mais. Campinas, São Paulo, Brasil, 2001-2002.

\begin{tabular}{lcccc}
\hline \multirow{2}{*}{ Faixa etária (anos) } & \multicolumn{2}{c}{ Masculino } & \multicolumn{2}{c}{ Feminino } \\
& Média & IC95\% & Média & IC95\% \\
\hline $18-29$ & 1,24 & $1,03-1,45$ & 1,32 & $1,08-1,56$ \\
$30-39$ & 1,21 & $1,07-1,35$ & 1,40 & $1,06-1,74$ \\
$40-49$ & 1,52 & $0,97-2,07$ & 1,86 & $1,45-2,27$ \\
$50-59$ & 2,03 & $1,27-2,79$ & 2,27 & $1,61-2,93$ \\
$60-69$ & 2,33 & $1,88-2,78$ & 2,36 & $2,14-2,58$ \\
70 ou mais & 2,92 & $2,48-3,37$ & 3,55 & $2,96-4,14$ \\
Total & 1,81 & $1,51-2,10$ & 1,96 & $1,77-2,15$ \\
\hline
\end{tabular}

IC95\%: intervalo de 95\% de confiança. 
A Tabela 2 apresenta a prevalência e razão de prevalência do uso de ao menos um medicamento, segundo as variáveis demográficas e socioeconômicas. Para o conjunto da população estudada a prevalência do consumo de medicamentos foi de $48,5 \%$. A prevalência foi maior entre as mulheres e nas faixas etárias acima de 40 anos, destacando-se a prevalência de $88,4 \%$ no grupo etário de 70 anos ou mais. Quanto à religião, após ajuste por idade e sexo, os evangélicos apresentaram maior consumo de medicamentos no limiar de significância estatística. Observa-se em relação às variáveis socioeconômicas que o uso de medicamentos esteve associado com escolaridade e renda per capita. A prevalência do uso de medicamentos foi menor entre os indivíduos com 8 anos ou mais de escolaridade (43,9\%) e maior nos indivíduos com renda per capita superior a 4 salários mínimos $(57,1 \%)$. Após o ajuste por idade e sexo, permaneceu com significância estatística apenas a variável renda, com a categoria maior que 4 salários mínimos apresentando uso mais elevado de medicamentos.

Na Tabela 3, é apresentada a utilização dos medicamentos segundo comportamentos relacionados à saúde e morbidade. Mostraram-se

Tabela 2

Prevalência e razão de prevalência (RP) da utilização de ao menos um medicamento, segundo variáveis demográficas e socioeconômicas da população de 18 anos ou mais. Campinas, São Paulo, Brasil, 2001-2002.

\begin{tabular}{|c|c|c|c|c|c|}
\hline Variáveis & n & Prevalência (\%) & Valor de $p$ * & RP ajustada por idade e sexo & Valor de $p$ \\
\hline Sexo & & & 0,000 & & \\
\hline Masculino & 481 & 36,8 & & 1,00 & \\
\hline Feminino & 460 & 59,2 & & $1,58(1,32-1,91)$ & 0,000 \\
\hline Total & 941 & 48,5 & & & \\
\hline Idade (anos) & & & 0,000 & & \\
\hline $18-29$ & 242 & 34,6 & & 1,00 & \\
\hline $30-39$ & 95 & 38,2 & & $1,08(0,74-1,52)$ & 0,673 \\
\hline $40-49$ & 101 & 53,2 & & $1,54(1,14-2,07)$ & 0,006 \\
\hline $50-59$ & 77 & 54,7 & & $1,65(1,13-2,43)$ & 0,012 \\
\hline $60-69$ & 240 & 73,0 & & $2,10(1,57-2,79)$ & 0,000 \\
\hline$\geq 70$ & 186 & 88,4 & & $2,43(1,79-3,30)$ & 0,000 \\
\hline Situação conjugal & & & 0,045 & & \\
\hline Casado/Unido & 563 & 46,2 & & 1,00 & \\
\hline Solteiro & 176 & 47,3 & & $1,24(0,96-1,61)$ & 0,092 \\
\hline Separado/Viúvo & 200 & 59,8 & & $0,93(0,78-1,10)$ & 0,402 \\
\hline Cor/Etnia & & & 0,063 & & \\
\hline Branca & 763 & 50,3 & & 1,00 & \\
\hline Preta/Parda & 168 & 39,8 & & $0,83(0,66-1,04)$ & 0,117 \\
\hline Religião & & & 0,103 & & \\
\hline Católico & 673 & 46,4 & & 1,00 & \\
\hline Evangélico & 135 & 60,2 & & $1,28(0,99-1,64)$ & 0,052 \\
\hline Outras e sem religião & 133 & 47,4 & & $1,12(0,88-1,43)$ & 0,328 \\
\hline \multicolumn{6}{|l|}{ Escolaridade (em anos) } \\
\hline $0-3$ & 208 & 59,9 & 0,007 & 1,00 & \\
\hline $4-7$ & 278 & 52,4 & & $1,06(0,86-1,31)$ & 0,522 \\
\hline 8 ou mais & 454 & 43,9 & & $1,06(0,83-1,36)$ & 0,596 \\
\hline Renda per capita (salários mínimos) ** & & & 0,046 & & \\
\hline$<1$ & 164 & 39,0 & & 1,00 & \\
\hline $1-4$ & 522 & 47,3 & & $1,20(0,89-1,61)$ & 0,214 \\
\hline$>4$ & 255 & 57,1 & & $1,41(1,03-1,94)$ & 0,033 \\
\hline
\end{tabular}

* Valor de $p$ do teste $\chi^{2}$;

** Salário mínimo vigente à época da pesquisa: março-abril/2001 = R\$ 151,00; maio/2001 a março/2002 = R\$ 180,00 e abril-setembro/2002 = R\$ 200,00. 
Prevalência e razão de prevalência (RP) da utilização de ao menos um medicamento, segundo comportamentos relacionados à saúde e morbidade da população de 18 anos ou mais. Campinas, São Paulo, Brasil, 2001-2002.

\begin{tabular}{|c|c|c|c|c|c|}
\hline Variáveis & n & Prevalência (\%) & Valor de $p^{*}$ & RP ajustada por idade e sexo & Valor de $p$ \\
\hline Tabagismo & & & 0,156 & & \\
\hline Fumante & 169 & 41,3 & & 1,00 & \\
\hline Não-fumante & 559 & 49,3 & & $0,87(0,68-1,10)$ & 0,237 \\
\hline Ex-fumante & 212 & 54,9 & & $1,03(0,81-1,31)$ & 0,772 \\
\hline Frequência do consumo de álcool & & & 0,003 & & \\
\hline Não ingere & 432 & 56,9 & & 1,00 & \\
\hline Menos de 1 vez por semana & 176 & 49,7 & & $0,96(0,77-1,19)$ & 0,749 \\
\hline Mais de 1 vez por semana & 322 & 38,2 & & $0,85(0,67-1,02)$ & 0,081 \\
\hline Dependência de álcool (CAGE) & & & 0,051 & & \\
\hline Não depende & 887 & 49,3 & & 1,00 & \\
\hline Depende & 44 & 29,4 & & $0,71(0,38-1,33)$ & 0,277 \\
\hline Prática de atividade física & & & 0,088 & & \\
\hline Não & 622 & 48,2 & & 1,00 & \\
\hline Sim & 319 & 49,1 & & $1,12(0,94-1,34)$ & 0,192 \\
\hline Número de doença crônica & & & 0,000 & & \\
\hline Nenhuma & 236 & 22,2 & & 1,00 & \\
\hline $1-2$ & 378 & 50,1 & & $1,98(1,50-2,61)$ & 0,000 \\
\hline 3 ou mais & 303 & 76,5 & & $2,63(1,93-3,59)$ & 0,000 \\
\hline Presença de morbidade nos últimos 15 dias & & & 0,000 & & \\
\hline Não & 784 & 45,4 & & 1,00 & \\
\hline Sim & 157 & 66,0 & & $1,29(1,15-1,44)$ & 0,000 \\
\hline Hospitalização no último ano & & & 0,987 & & \\
\hline Nenhuma & 844 & 48,5 & & 1,00 & \\
\hline 1 ou mais & 97 & 48,6 & & $0,94(0,73-1,22)$ & 0,663 \\
\hline Transtorno mental comum & & & 0,001 & & \\
\hline Não & 754 & 45,5 & & 1,00 & \\
\hline Sim & 157 & 62,1 & & $1,19(1,00-1,40)$ & 0,042 \\
\hline
\end{tabular}

* Valor de $p$ do teste $\chi^{2}$

associadas ao uso de medicamentos as variáveis: frequência de consumo de bebida alcoólica, número de doenças crônicas, morbidade de 15 dias e transtorno mental comum. Após o ajuste por idade e sexo, apenas as variáveis número de doenças crônicas, presença de morbidade nos últimos 15 dias e transtorno mental comum permaneceram associadas ao uso de medicamentos.

No modelo final permaneceram significativamente associadas ao uso de medicamentos as categorias: sexo feminino, idade entre 40-59 anos e 60 e mais, morbidade referida nos últimos 15 dias e número de doenças crônicas (uma e duas, três ou mais) apresentadas na Tabela 4. A associação com a religião desaparece quando foram introduzidas no modelo as variáveis número de doenças crônicas e morbidade de 15 dias, indi- cando que estas variáveis são mediadoras da sua influência sobre o uso de medicamentos.

Observa-se na Tabela 5 que os grupos de medicamentos mais utilizados foram os que atuam no sistema cardiovascular (16,8\%) e sistema nervoso (16,3\%). Entre os fármacos mais consumidos destacaram-se: Hidroclorotiazida, Captopril, Enalapril, Dipirona e o Ginkgo biloba. Os medicamentos fitoterápicos estiveram entre os mais utilizados pela população estudada.

\section{Discussão}

Nos últimos anos tem crescido o interesse em avaliar o uso de medicamentos e os fatores determinantes deste uso pela população. $\mathrm{O}$ interesse justifica-se pelo consumo crescente de 
Modelo de regressão múltipla de Poisson para uso de ao menos um medicamento, na população de 18 anos ou mais. Campinas, São Paulo, Brasil, 2001-2002.

\begin{tabular}{|c|c|c|c|}
\hline Variável & RP & IC95\% & Valor de $p$ \\
\hline \multicolumn{4}{|l|}{ Sexo } \\
\hline Masculino & 1,00 & & \\
\hline Feminino & 1,38 & $1,14-1,67$ & 0,002 \\
\hline \multicolumn{4}{|l|}{ Idade (anos) } \\
\hline $18-39$ & 1,00 & & \\
\hline $40-59$ & 1,30 & $1,07-1,58$ & 0,010 \\
\hline 60 e mais & 1,66 & $1,40-1,97$ & 0,000 \\
\hline \multicolumn{4}{|c|}{ Número de doenças crônicas } \\
\hline Nenhuma & 1,00 & & \\
\hline Uma a duas & 1,98 & $1,50-2,61$ & 0,000 \\
\hline Três ou mais & 2,61 & $1,93-3,53$ & 0,000 \\
\hline \multicolumn{4}{|c|}{ Morbidade nos últimos 15 dias } \\
\hline Não & 1,00 & & \\
\hline Sim & 1,22 & $1,06-1,40$ & 0,000 \\
\hline
\end{tabular}

IC95\%: intervalo de 95\% de confiança; RP: razão de prevalência.

Tabela 5

Prevalência de uso de medicamentos na população de 18 anos ou mais segundo a classificação Anatomical Therapeutic Chemical Index (1o e 5o * níveis da ATC). Campinas, São Paulo, Brasil, 2001-2002.

\begin{tabular}{lc}
\hline Classes terapêuticas & $\%$ \\
\hline Trato alimentar e metabolismo (A) & 9,15 \\
Sangue e órgãos formadores de sangue (B) & 2,90 \\
Ácido acetil salićlico usado como antiagregador plaquetário & 2,34 \\
Sistema cardiovascular (C) & 16,82 \\
Hidroclortiazida & 2,76 \\
Captopril & 2,75 \\
Enalapril & 1,87 \\
Dermatológicos (D) & 1,32 \\
Sistema geniturinário e hormônios sexuais (G) & 6,20 \\
Preparados para o sistema hormonal (H) & 2,50 \\
Levotiroxina & 1,62 \\
Anti-infecciosos gerais para uso sistêmico (J) & 0,60 \\
Antineoplásicos e imunomoduladores (L) & 0,62 \\
Sistema músculo-esquelético (M) & 5,67 \\
Diclofenaco & 2,82 \\
Sistema nervoso (N) & 16,34 \\
Dipirona & 6,88 \\
Ginkgo biloba & 1,34 \\
Sistema respiratório (R) & 3,10 \\
Orgãos sensoriais (S) & 0,44 \\
Outros medicamentos & 6,69 \\
Fitoterápicos & 2,94 \\
\hline
\end{tabular}

* Mais frequentes no 5 o nível. 
medicamentos nos diferentes segmentos sociais, pelo alto investimento dos governos para ampliação do acesso aos medicamentos e pela complexidade do mercado farmacêutico em relação a número, variedade e qualidade de produtos, entre outros.

O presente estudo aponta uma prevalência no consumo de medicamentos pela população de 18 anos ou mais do Município de Campinas de $48,5 \%$, o que se mostrou inferior à maioria dos achados de outros estudos nacionais 2,3,4,17 e internacionais 18 que utilizaram períodos recordatórios maiores. Os estudos realizados em Pelotas, Rio Grande do Sul ${ }^{3}$, e Fortaleza, Ceará 4 , que incluíram a população adulta, utilizaram como período recordatório 15 dias e encontraram, respectivamente, as prevalência de uso de medicamentos de 65,9\% e 49,7\%. Na Espanha, Sans et al. 18 identificaram uma prevalência de uso entre adultos de $67 \%$. Outros estudos nacionais que utilizaram o período recordatório de 3 dias apresentaram prevalência de uso inferiores, $33,9 \% 19$ e $41,2 \% 20$, quando comparados ao presente trabalho.

O número médio de medicamentos utilizados foi de 1,88, semelhante ao encontrado em Araraquara, São Paulo $(1,8)$ 21, Pelotas $(1,5)^{3}$ e Fortaleza $(1,9) 4$. Observa-se que há um crescimento desse número com o aumento da idade, em especial no sexo feminino, o que corrobora com a literatura consultada 19,20.

O consumo crescente de medicamentos com o aumento da idade encontrado no estudo é evidenciado na literatura nacional 2,3,22,23, destacando um crescimento especial na faixa etária acima de 40 anos. Uma explicação para essa associação positiva entre idade e o elevado consumo de medicamentos é a maior prevalência das morbidades nas idades mais avançadas 4 .

Observa-se um maior consumo entre as mulheres em todas as faixas etárias, o que corrobora com a literatura 4,18,19,20,24. A diferença entre as mulheres e os homens tem sido explicada por aspectos socioculturais e biológicos que favoreceriam maior adoecimento, maior autocuidado, maior busca por serviços de saúde e maior exposição a medicamentos entre as mulheres 25 . Os serviços de saúde em geral prestam mais assistência ao atendimento das mulheres com programas específicos, como o pré-natal, prevenção de câncer do colo uterino e mama, entre outros 3,18 .

A renda familiar aparece associada com o consumo de medicamentos no presente estudo. Verifica-se que indivíduos com renda per capita superior a 4 salários mínimos consumiram mais medicamentos em relação aos com renda inferior. Tal resultado aponta que o poder aquisitivo do indivíduo é um fator preditivo para o uso de medicamentos, o que é verificado na literatura 3,4 . Reconhece-se que seguimentos de pior nível socioeconômico têm menos acesso aos serviços de saúde e aos medicamentos em geral. Segundo a Organização Mundial da Saúde (OMS), um terço da população mundial não tem acesso aos medicamentos essenciais 26 , considera-se que no Brasil uma parcela representativa da população depende do Sistema Único de Saúde (SUS) para aquisição dos medicamentos 4 . Nesse contexto, é importante destacar que as Políticas Nacionais de Medicamentos e assistência Farmacêutica objetivam o acesso e a promoção do uso racional dos medicamentos, e que nos últimos anos várias ações coordenadas pelo Ministério da Saúde têm possibilitado o avanço da implementação e qualificação desta assistência no país, como a ampliação do financiamento público para garantir a aquisição dos medicamentos que estão selecionados e incorporados no SUS 27.

Verifica-se que existe associação entre o número de doenças crônicas e morbidade referida nos 15 dias e o uso de medicamentos, o que era esperado e observado em outros estudos nacionais, pois as pessoas nestas condições de saúde buscam mais os serviços e o medicamento é uma das intervenções terapêuticas utilizadas 2,4 .

Em relação à religião observou-se maior consumo de medicamentos pelos evangélicos, sendo que esta associação perde a significância quando foram introduzidas as variáveis de morbidade no modelo. Estudo realizado no projeto SABE (Saúde, Bem-Estar e Envelhecimento) 28, com a população idosa do Município de São Paulo, não encontrou diferença significativa de uso de medicamentos segundo religião. Não foi encontrado outro estudo que avaliou o uso de medicamentos associado à religião. Lima et al. 29, analisando a qualidade de vida relacionada à saúde em idosos, observaram escores mais baixos nos evangélicos relativamente ao segmento de católicos. Estudos apontam para o pior nível socioeconômico entre os evangélicos 24 e tendência de os indivíduos com o menor nível socioeconômico apresentarem mais morbidades 30 .

Uma das limitações do presente trabalho é que por ser um estudo transversal não permite a identificação da relação causa e efeito. Em relação aos aspectos metodológicos utilizados, cabe considerar alguns fatores que influenciam os resultados, como o período recordatório utilizado para identificar o consumo de medicamentos da população estudada.

Segundo Bertoldi et al. 5 , não existe consenso na literatura sobre o período recordatório ideal para investigar o uso de medicamentos na população, pois a seleção do período depende do que 
se pretende que seja recordado e da capacidade dos indivíduos em lembrar. Sabe-se que a prevalência do uso de medicamentos é dependente da definição desse período, e uma das principais questões metodológicas relacionadas é a dificuldade de recordação com precisão das informações, que é influenciada pelo aumento da idade e do número de medicamentos utilizados no período. Outro aspecto a ser considerado é a precisão da informação que pode depender do estado de saúde em que o indivíduo se encontra. Além desses pontos abordados, a dificuldade de recordação pode ser acentuada quanto maior o período a ser lembrado, o que pode acarretar numa subnotificação de alguns tipos de medicamentos e uma subestimação da sua utilização ${ }^{3}$.

Kohn et al. 31 , em um estudo multicêntrico realizado em diferentes países, relataram que um período recordatório entre dois dias, que foi utilizado para estimar a prevalência do uso de medicamentos no referido estudo, facilitou a lembrança de seus entrevistados, gerando dados fidedignos. Nesse contexto, o presente estudo optou por um período recordatório mais curto, visando a garantir a qualidade da informação referida, mesmo que dificultasse a comparação com outros estudos nacionais.

No modelo final verifica-se que permaneceram associados ao uso de medicamentos sexo, idade, número de doenças crônicas e morbidade referida nos últimos 15 dias, evidenciando que estas variáveis estão independentemente associadas ao uso de medicamentos nessa população.

A classificação dos medicamentos, segundo a ATC, permite identificar que o consumo da população de 18 anos ou mais de Campinas é maior entre os medicamentos que atuam no sistema cardiovascular e sistema nervoso, o que é encontrado na literatura nacional 1,7,32,33.

Esse maior consumo dos medicamentos que atuam no sistema cardiovascular pode estar relacionado ao fato das doenças cardiovasculares, entre elas a hipertensão arterial, terem alta prevalência, em especial na população idosa. Os medicamentos mais utilizados nesse grupo terapêutico foram o Captopril, a Hidroclorotiazida e o Enalapril como foi também observado por
Arrais ${ }^{1}$ em Fortaleza. Verificou-se um alto consumo dos analgésicos, destacando-se os antipiréticos, em especial a Dipirona. Esse achado já tem sido evidenciado desde a década de 80 no Brasil e em estudos internacionais, como sendo uma das classes farmacológicas de maior consumo pela população 1 . Em relação aos medicamentos que atuam no sistema nervoso o presente trabalho encontrou valores inferiores a outros estudos nacionais $1,3,19$.

A utilização de medicamentos fitoterápicos constitui um dos principais grupos de consumo pela população de Campinas. Esse achado pode estar relacionado ao fato de o Município de Campinas ter implementado uma Política $\mathrm{Mu}$ nicipal de Fitoterapia que incentiva o uso dessa alternativa terapêutica e oferece o acesso a esses medicamentos nas farmácias municipais. $\mathrm{O}$ maior consumo do Ginkgo biloba também foi encontrado em estudo realizado em São Paulo por Pelicione 19, estando entre os 21 medicamentos mais utilizados.

\section{Conclusão}

A prevalência do uso de medicamentos entre a população de 18 anos ou mais, residente em Campinas, apresentou-se inferior à maioria dos estudos e o maior uso esteve associado ao sexo feminino, idade acima de 40 anos, morbidade referida nos últimos 15 dias e número de doenças crônicas. As associações entre o consumo de medicamentos com fatores demográficos e de morbidade apontam a importância do uso de medicamentos no processo saúde/doença. Os inquéritos de saúde locais tornam-se necessários para dar conta do conhecimento sobre a utilização de medicamentos pelos diferentes segmentos sociais da população, sobre o tipo de medicamento consumido, o perfil demográfico de quem os consome, entre outras informações. Essas informações permitem aos gestores a melhor compreensão do uso de medicamentos pela população, possibilitando intervenções mais direcionadas nas práticas de saúde, visando à garantia do acesso e o correto uso dos medicamentos. 


\section{Resumo}

Este artigo analisa a prevalência da utilização de medicamentos segundo variáveis demográficas, socioeconômicas e de comportamentos relacionados à saúde, identificando fatores associados ao uso por meio de estudo transversal de base populacional, com 941 pessoas de 18 anos ou mais residentes em Campinas, São Paulo, Brasil. A amostragem foi realizada em múltiplos estágios, estratificada e por conglomerados. Utilizou-se o teste qui-quadrado, foram estimadas as razões de prevalência ajustadas por sexo e idade e os respectivos IC95\%. Desenvolveu-se modelo de regressão múltipla de Poisson ficando associados ao uso: sexo feminino, idade de 40 anos e mais, morbidade referida nos últimos 15 dias e número de doenças crônicas. Os medicamentos mais consumidos foram para os sistemas cardiovascular e nervoso, e os fitoterápicos. A prevalência de uso de medicamentos em Campinas encontrou-se inferior à maioria dos estudos. Por meio de inquéritos de saúde locais espera-se conhecer o perfil de uso dos medicamentos pela população e garantir intervenções mais direcionadas para a Política de Assistência Farmacêutica.

Uso de Medicamentos; Farmacoepidemiologia; Prevalência

\section{Colaboradores}

K. S. Costa participou da revisão da literatura, análise e interpretação dos dados e redação do artigo. M. B. A. Barros orientou a proposta do artigo, a análise dos dados, redação do artigo e coordenou a pesquisa de campo. P. M. S. B. Francisco orientou a proposta do artigo, a análise dos dados e a redação do artigo. C. L. G. César, M. Goldbaum e L. Carandina coordenaram a pesquisa de campo e contribuíram com a revisão do artigo.

\section{Agradecimentos}

À Fundação de Amparo à Pesquisa do Estado de São Paulo (Projeto de Políticas Públicas, processo $n^{\circ}$. 88/14099) e à Secretaria Estadual de Saúde de São Paulo pelo financiamento do trabalho de campo. À Secretaria de Vigilância em Saúde do Ministério da Saúde pelo suporte financeiro para a análise dos dados por meio do Centro Colaborador em Análise de Situação de Saúde da Universidade Estadual de Campinas.

\section{Referências}

1. Arrais PSP. Medicamentos: consumo e reações adversas - um estudo de base populacional. Fortaleza: Edições UFC; 2009.

2. Loyola Filho AI, Uchoa E, Guerra HL, Firmo JOA, Lima-Costa MF. Prevalência e fatores associados à automedicação: resultados do projeto Bambuí. Rev Saúde Pública 2002; 36:55-62.

3. Bertoldi AD, Barros AJD, Hallal PC, Lima RC. Utilização de medicamentos em adultos: prevalência e determinantes individuais. Rev Saúde Pública 2004; 38:228-38.

4. Arrais PSP, Brito LL, Barreto ML, Coelho HLL. Prevalência e fatores determinantes do consumo de medicamentos no Município de Fortaleza, Ceará, Brasil. Cad Saúde Pública 2005; 21:1737-46.
5. Bertoldi AD, Barros AJD, Wagner A, Rooss-Degnan D, Hallal PC. A descriptive review of the methodologies used in household surveys on medicine utilization. BMC Health Serv Res 2008; 8:222.

6. Rozenfeld S, Valente J. Estudos de utilização de medicamentos - considerações técnicas sobre coleta e análise de dados. Epidemiol Serv Saúde 2004; 13:115-23.

7. Barros MBA. Inquéritos domiciliares de saúde: potencialidades e desafios. Rev Bras Epidemiol 2008; 11 Suppl 1:6-19.

8. Castro SS, Pellicioni AF, Cesar CLG, Carandina L, Barros MBA, Alvez MCGP, et al. Uso de medicamentos por pessoas com deficiência em áreas do Estado de São Paulo. Rev Saúde Pública 2010; 44:601-10. 
9. Paniz VMV, Fassa AG, Facchini LA, Bertoldi AD, Piccini RX, Tomasi E, et al. Acesso a medicamentos de uso contínuo em adultos e idosos nas regiões Sul e Nordeste do Brasil. Cad Saúde Pública 2008; 24:267-80.

10. Ribeiro AQ, Rozenfeld S, Klein CH, César CC, Acurcio FA. Inquérito sobre o uso de medicamentos por idosos aposentados, Belo Horizonte, MG. Rev Saúde Pública 2008; 42:724-32

11. César CLG, Carandina L, Alves MCGP, Barros MBA, Goldbaum M. Saúde e condição de vida em São Paulo. Inquérito multicêntrico de saúde no Estado de São Paulo ISA-SP. São Paulo: Faculdade de Saúde Pública, Universidade de Saúde Pública; 2005.

12. Alves MCGP. Plano de amostragem. In: Barros MBA, César CLG, Carandina L, Goldbaum M, organizadores. As dimensões da saúde: inquérito populacional em Campinas. São Paulo: Editora Hucitec; 2008. p. 46-55.

13. Dicionário de Especialidades Farmacêuticas: DEF 2001/02. 30a Ed. Rio de Janeiro: Editora Publicações Científicas; 2000.

14. WHO Collaborating Centre for Drug Statistics Methodology. Guidelines for ATC classification and DDD assignment. 3rd Ed. Oslo: World Health Organization; 2000.

15. Bisson J, Nadeau L, Demers A. The validity of the CAGE scale to screen for heavydrinking and drinking problem in a general population survey. Addiction 1999; 94:715-22.

16. Mari JJ, Williams P. A validity study of a psychiatric screening questionnaire (SQR-20) in primary care in the city of São Paulo. Br J Psychiatry 1986; 148:23-6.

17. Barros MBA. Saúde e classe social: um estudo sobre morbidade e consumo de medicamentos, 1983, Ribeirão Preto [Tese de Doutorado]. Ribeirão Preto: Faculdade de Medicina de Ribeirão Preto, Universidade de São Paulo; 1983.

18. Sans S, Paluzie G, Puig T, Balañá L, Balaguer-Vintró I. Prevalencia del consumo de medicamentos en la población adulta de Cataluña. Gac Sanit 2002;16:121-30.

19. Pelicione AF. Padrão de consumo de medicamentos em duas áreas da Região Metropolitana de São Paulo, 2001-2002 [Dissertação de Mestrado]. São Paulo: Faculdade de Saúde Pública, Universidade de Saúde Pública; 2005.

20. Perrone AMF, Molina MC, Bertonha MEAM, Nativio J, Barros MBA. Uso de medicamentos. In: Barros MBA, César CLG, Carandina L, Goldbaum M, organizadores. As dimensões da saúde: inquérito populacional em Campinas. São Paulo: Editora Hucitec; 2008. p. 218-29.
21. Simões MJS, Farache Filho A. Consumo de medicamentos em região do Estado de São Paulo (Brasil), 1985. Rev Saúde Pública 1988; 22:494-9.

22. Coelho Filho JM, Marcopito LF, Castelo A. Perfil de utilização de medicamentos por idosos em área urbana do Nordeste do Brasil. Rev Saúde Pública 2004; 38:557-64.

23. Flores VB, Benvegnú LA. Perfil de utilização de medicamentos em idosos da zona urbana de Santa Rosa, Rio Grande do Sul, Brasil. Cad Saúde Pública 2008; 24:1439-46

24. Floriano PJ, Dalgalarrondo P. Saúde mental, qualidade de vida e religião em idosos de um Programa de Saúde da Família. J Bras Psiquiatr 2007; 56 162-70.

25. Santos DB, Barreto ML, Coelho HL. Utilização de medicamentos e fatores associados entre crianças residentes em áreas pobres. Rev Saúde Pública 2009; 43:768-78.

26. Ministério da Saúde/Organização Pan-Americana da Saúde/Organização Mundial da Saúde. Avaliação da assistência farmacêutica no Brasil: estrutura, processo e resultados. Brasília: Ministério da Saúde; 2005.

27. Departamento de Assistência Farmacêutica e Insumos Estratégicos, Secretaria de Ciência, Tecnologia e Insumos Estratégicos. Da excepcionalidade às linhas de cuidado: o Componente Especializado da Assistência Farmacêutica. Brasília: Ministério da Saúde; 2010.

28. Carvalho MFC. A polifarmácia em idosos no $\mathrm{Mu}$ nicípio de São Paulo - Estudo SABE - Saúde, BemEstar e Envelhecimento [Dissertação de Mestrado]. São Paulo: Faculdade de Saúde Pública, Universidade de São Paulo; 2007.

29. Lima MG, Barros MBA, César CLG, Goldbaum M Carandina L, Ciconelli RM. Health related quality of life among the elderly: a population-based study using SF-36 survey. Cad Saúde Pública 2009; 25:2159-67.

30. Barros MBA, César CLG, Carandina L, Torre GD. Desigualdades sociais na prevalência de doenças crônicas no Brasil, PNAD-2003. Ciênc Saúde Coletiva 2006; 11:911-26.

31. Kohn R, White KL. Health care: an international study. London: Oxford University Press; 1976.

32. Danhier A, Brieva J, Villegas G, Yates T, Pérez H, Boggiano G. Utilización de medicamentos en una población urbana. Rev Med Chil 1991; 119:334-7.

33. Lima MCP, Menezes PR, Carandina L, César CLG, Barros MBA, Goldbaum M. Transtornos mentais comuns e uso de psicofármacos: impacto das condições socioeconômicas. Rev Saúde Pública 2008; 42:717-23.

Recebido em 15/Ago/2010

Versão final reapresentada em 25/Jan/2011

Aprovado em 01/Fev/2011 